\title{
Single-subject statistical analysis of computer-assisted biofeedback
}

\author{
GEORGE ALAN BROWN and FRANCISCO I. PEREZ \\ Baylor College of Medicine, Houston, Texas
}

\begin{abstract}
Single-subject statistics were used to analyze physiological data [electromyographic (EMG) temperature] recorded from two patients receiving computer-assisted biofeedback treatment in a clinic. When the data were analyzed by an ANOVA method or a regression technique, the results demonstrated that both patients learned to significantly reduce EMG levels. The rationale of using single-subject statistics is discussed, and the applicability of the statistical technique to the clinic population is illustrated.
\end{abstract}

Biofeedback has been used to treat various disorders since 1969, when N. Miller and J. Kamiya independently demonstrated that the autonomic nervous system could be modified by instrumental conditioning (Kewman \& Roberts, 1983). Some of these clinically relevant disorders include tension headaches, migraine headaches, anxiety, functional diarrhea, Raynaud's disease, colitis, essential hypertension, asthma, sinus tachycardia, premature ventricular contractions, and fecal incontinence, to name but a few (Kolotkin, Billingham, \& Feldman, 1981). The rationale for using biofeedback in these various disorders is theoretically sound and somewhat substantiated by the success of numerous single-case reports. However, recent reviews of the efficacy for clinical applications of biofeedback have failed to demonstrate that biofeedback directly mediates target symptoms or is superior to other treatments (Kewman \& Roberts, 1983).

The inability of seemingly well-controlled experiments to replicate findings across studies may be due to problems in experimental design, instrumentation, treatment protocols, and methods of data analysis (Kolotkin et al., 1981). The failure to replicate significant biofeedback effectiveness cannot be construed as the "technique biofeedback does not work." In fact, it does tell us that perhaps we need to reassess our tools for measuring and evaluating biofeedback outcome.

Armed with multichannel physiographs and computers, laboratory workers have devised controlled protocols, elaborate designs, and statistical analyses to investigate the variables at work. On the other hand, clinics or small private practices have relied on techniques developed in the laboratory but often have used these without control or precision. The very nature of biofeedback in the clinic is sometimes different from that in the research laboratory. Kewman and Roberts (1983) stated that "the purpose of scientific study is the application of the scientific

We gratefully acknowledge the assistance of Michael Yost and Fred Bremner for their assistance in the design and analysis of this study. Requests for reprints should be sent to Francisco I. Perez, Behavioral Medicine Consultants, 6560 Fannin, Suite 1224, Houston, TX 77030. method in order to understand, predict, and control observable phenomenon. The primary goal of therapy is to change the behavior of patients. Experiments need to be designed that delineate the essential ingredients in biofeedback treatment and how they may be manipulated most effectively in order to produce clinical change"' (p. 489). Steiner and Dince (1983) commented on why some studies do not demonstrate an effect when positive changes are clinically noticeable. True sophistication in biofeedback research requires an appropriate synthesis of research and clinical practice.

This synthesis of research and clinical practice may indeed yield some answers. The synthesis is made possible by the advent of the small biofeedback computer and new ways of looking at behavior-change experimental designs (Hersen \& Barlow, 1976). The small biofeedback computer is affordable to even private practitioners. Brown and Perez (1985) offered the following advantages of computer-assisted biofeedback treatment: (1) The computer introduces precision in data acquisition; (2) structured treatment protocols may be defined and replicated easily across investigators; (3) the computer's adaptive capacity makes shaping responses according to the laws of operant conditioning easy; (4) the computer has the ability to analyze several sources of information and to determine patterns of complex physiological responding; and (5) the computer offers many advantages for data analysis and storage.

The introduction of the computer into the clinical setting may help reduce a certain amount of variability and make it possible to investigate new methods of data analysis. Biofeedback is ideally suited to the process-oriented, single-case study (Ax, 1981). Biofeedback monitoring, over time, should provide more knowledge and prediction of individual response and learning than can be arrived at through the usual normative paradigm (Johnston \& Pennypacker, 1980). However, Bandeira, Bouchard, and Granger (1982), in a review of 165 studies of cardiac biofeedback data, found that only $5 \%$ adopted an individual design. In addition, most single-subject biofeedback research has been summarized using descriptive 
statistics. Inferential statistics of single-subject data have been given very little attention, probably because early laboratory research yielded such clear-cut distinctions that users did not have to resort to tests of statistical significance (Kelly, McNeil, \& Newman, 1973). At present, clinical research in biofeedback is conducted in settings in which control of extraneous variables is difficult to achieve. As a consequence, data often fail to exhibit the clear magnitude of effect that one may find in the laboratory.

Another reason to use the single-subject design is that physiological responses are unique for each individual (Carroll \& Phys-Davies, 1979). Several investigators have demonstrated that the vast majority of the autonomic response has a small correlation between subjects, but shows considerable intrasubject consistency, that is, a pattern of autonomic reactivity that characterizes the individual. This would favor the use of individual rather than group data designs. Numerous investigators have demonstrated the rationale of applying multivariate statistics to single subjects (Hersen \& Barlow, 1976; Kelly et al., 1973; Kratochwill, 1978; Shine, 1982). The applicability of multivariate data techniques to the EEG of a single subject has been shown in several studies (Bremner, McKenzie, Yost, \& Eddy, 1982; Bremner, Yost, \& McKenzie, 1982; Brown, 1982). These investigators demonstrated consistency in a single subject's EEG spectra under a particular treatment condition across days. They also found significant differences in the EEG spectra between treatments. The data were also analyzed as a group design. The results then showed no significant differences in EEG between treatment conditions when merged across subjects. The differences that were found between conditions were "washed out" in the merging of the subjects. It is in conditions like these that the singlesubject design is powerful in detecting differences when differences actually exist.

After the successful development of single-subject statistical applications in the laboratory, we decided to assess the applicability of the design to the clinical setting using a computer-assisted biofeedback procedure.

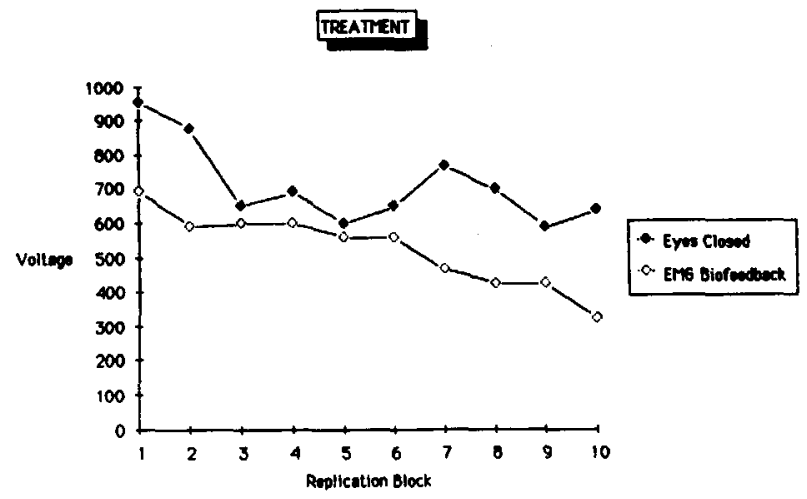

Figure 1. Mean EMG levels by treatment across days for Experiment 1.

\begin{tabular}{lrcc}
\multicolumn{5}{c}{ Table 1 } \\
ANOVA & & \\
\hline \multicolumn{1}{c}{ Source } & df & F & p $<$ \\
\hline Condition & 1 & 8.45 & $.0094^{*}$ \\
Observation & 19 & 1.88 & $.0182^{*}$ \\
Condition $\times$ Observation & 19 & 1.53 & n.s. \\
\hline
\end{tabular}

$*_{p}<.005$

\section{EXPERIMENT 1}

\section{Method}

Subject. The patient was a 38-year-old male who presented a history of episodic stress disorders spanning the last several years. Symptoms included nervousness, restlessness, gastrointestinal distress, and occasional headaches. The patient reported that these symptoms became worse under stress. A medical examination revealed no physical problems except a mild increase in heart rate.

Apparatus. The patient was seated in a comfortable recliner and instrumented for EMG recording with three silver/silver-chloride electrodes. The recording site covered a large area of the head and neck muscles. Active electrodes were placed on the right frontalis and the right cervical muscles. Ground was referenced 2 in. from the cervical electrode. Electrode impedance was maintained below $20 \mathrm{kohms}$. The electrode leads were attached to a remote preamplifier for signal amplification before being fed into a Cyborg Biolab System with an M130 differential amplifier. This amplifier has a range of 1 to $100 \mu \mathrm{V}$ RMS, a bandpass filter of 100 to $250 \mathrm{~Hz}$, and a time constant of $.022 \mathrm{sec}$. The signal was amplified and conditioned before being output to a 12-bit A/D converter contained in a $128 \mathrm{~K}$ Apple //e computer. Auditory feedback consisted of an analog tone that varied in pitch corresponding to the rise and fall of the EMG. The feedback was presented to the patient via an external speaker placed at a distance of 24 in. from the patient. The raw EMG was monitored by the experimenter by means of an oscilloscope and by computer-generated graphic displays of the digital $A / D$ values.

Procedure. The patient completed two experimental tasks. During the "eyes closed" condition, the subject sat quietly and attempted to relax as completely as possible. During "EMG biofeedback" training, the patient attempted to lower the pitch of the feedback tone by relaxing his muscles. In both conditions, he was instructed to close his eyes.

Data collection. The Apple //e controlled the auditory feedback training, data collection, and timing functions. The EMG was sampled once every .1 sec to provide auditory feedback during the biofeedback training. During data collection for both conditions, the $\mathrm{A} / \mathrm{D}$ sampling rate was 256 samples/sec. As soon as a trial had been completed, the data values in the Apple's main memory were transferred to an auxiliary bank of memory (128K) contained on the 80-column video card. This memory served as a high-speed "ram disk" to circumvent the slowness of conventional mechanical disk drives. The computer 
vaired the period between samples during each condition. Twenty seconds of data completed a presentation. After a variable rest period, the next presentation commenced. After 10 presentations or $400 \mathrm{sec}$ of data, the data were downloaded to floppy disks for storage. The Apple //e performed off-line analysis of each set of 256 data points (second). Data were then transmitted via a 300-baud modem to a mainframe computer at Rice University for further statistical analyses.

\section{Results}

A plot of the mean EMG levels for the "eyes closed" and the "EMG biofeedback" conditions across replication blocks is shown in Figure 1. The mean EMG voltage decreases across replication blocks. This correlates with the patient's subjective feelings of muscular relaxation. Although mean voltages of the "eyes open" condition do appear to decrease, the mean values are higher than those of the EMG condition. The data were submitted to a test of autocorrelation to check for dependency in the observations. After removing dependency effects, the data were analyzed by an ANOVA technique on the residual of the $2 \times 10$ design. As can be seen in Table 1 , significant differences were observed between conditions $[F(1)=8.45, p<.0094]$ and within observations $[F(19)=1.88, p<.0182]$. The interaction of condition and observation was not significant. A Scheffe's test was performed on the data, and the results revealed that the "eyes closed" residual was higher on average than expected and that the EMG feedback residual was lower than expected. A Newman-Keuls test demonstrated that the last observation in the set was different from all others.

\section{EXPERIMENT 2}

\section{Method}

Subject. The patient was a 21-year-old female who had been referred to the clinic for migraine headaches and who continues to receive treatment.

Apparatus. The experimental apparatus was the same as that in Experiment 1, with the following exceptions.

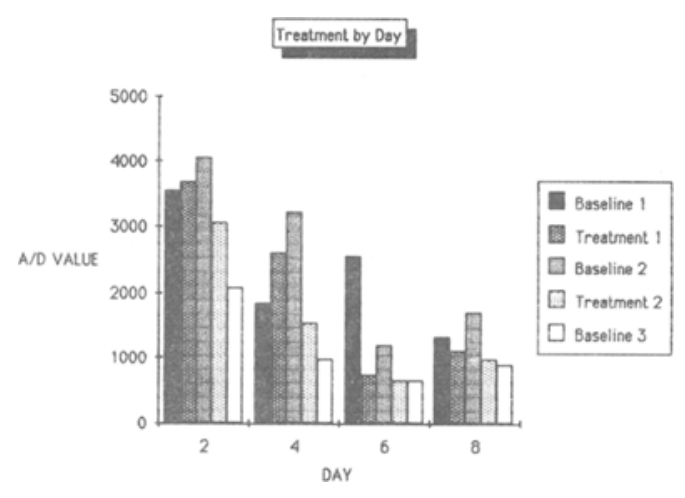

Figure 2. Mean EMG levels by treatment across days for Experiment 2.

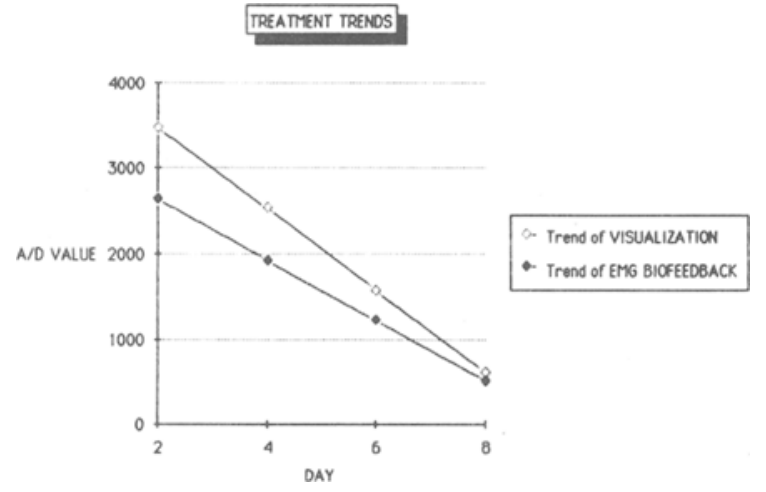
2.

Figure 3. Linear trends of treatments across days for Experiment

The patient was instrumented with EMG electrodes to measure muscle activity and a thermistor to measure peripheral skin temperature. Placement of the thermistor was on the fifth digit of the nondominant hand, according to accepted placement for thermal biofeedback. The patient's EMG was recorded by an Autogen 1700 EMG biofeedback instrument. The output of the instrument was connected to an M272 isolated instrument interface (Cyborg Corp.). Peripheral temperature was recorded by an M120 thermal module (Cyborg Corp.) The outputs of the temperature and EMG were directed to a 12-bit A/D converter controlled by the Apple //e computer used in the above experiment.

Procedure. The patient practiced a visual imagery technique and EMG biofeedback training. The patient's baseline was measured before each task and immediately after the second treatment condition.

Data collection. Measures of EMG and temperature were recorded and A/D converted at a rate of $256 \mathrm{samples} / \mathrm{sec}$. Twelve seconds of data were collected under each condition in two 6-sec blocks. There were 3,072 samples per condition on a given day. The digitized data were stored on a Corvus 20MB Winchester disk drive system. At the end of a day, the data were downloaded to floppy disks for backup. Data (as text files) were sent by the Apple //e computer to an IBM PC via a serial hardwire hookup. The IBM PC performed data transformations and then uploaded the data to an IBM 3031 mainframe at Trinity University for storage and later statistical analyses.

\section{Results}

It is apparent from Figure 2 that the overall EMG levels decreased over days. This corresponded to the patient's subjective reports of decreased headache symptoms. If the trends of the means for each of the treatments (visualization and biofeedback) are plotted, both treatments produce a significant negative trend (visualization, $R=.8826$, slope $=-477, \mathrm{p}<.001 ;$ biofeedback, $\mathrm{R}=.7949$, slope $=-355, \mathrm{p}<.001)$. This is demonstrated in Figure 3. It appears that EMG biofeedback provides more reduction in muscle activity in the earlier days of treatment. 


\section{DISCUSSION}

Both patients were able to reduce EMG muscle activity with biofeedback and visual imagery techniques. Furthermore, when analyzed by single-subject statistics, significant decreases are obtained in EMG muscle activity. Figures 1 and 2 demonstrate that, as treatment progresses, the patient achieves an overall reduction in EMG muscle activity. In addition to reducing muscle activity, both subjects reported a reduction in symptomatology. Note that the baseline condition between $\mathrm{T} 1$ and $\mathrm{T} 2$ is elevated on particular days. We discovered an artifact in our procedure associated with the experimenter's entering the room after the baseline to adjust the instrumentation. Upon questioning the patient, we decided that the elevations in EMG at this point might have been due to anticipatory responses from the subject before the experimenter entered the room.

We presented a rationale for the use of single-subject statistics in view of the problems inherent in clinical research, in which there are certain objections to using traditional group designs. It is difficult to find large numbers of patients who are homogeneous for a particular disorder. In group designs, averaging of results obscures individual data, because patients are rarely homogeneous. There is often a problem in generalizing the findings. Since results from group studies do not reflect changes in individual patients, these findings are not readily applicable for the practicing clinician, who cannot determine which particular patient characteristics are correlated with improvement. Pre and post measures do not examine the clinical course of a specific patient during treatment, so valuable data which could be used in the treatment process is lost.

In clinical applications of biofeedback, there is usually no return to baseline. In the training of physiological responses, this is a desirable outcome and lends itself to a multiple baseline technique.

One of the strong points of single-subject design is that the patient experiences all conditions within a day and across replications (days). Kazdin (1976) cited several situations in intrasubject replication research that many investigators believe are suitable for single subject statistics: (1) failure to establish stable baseline; (2) investigations in "new" areas of research; and (3) situations in which intrasubject variability is relatively large, perhaps due to the lack of experimental control over the situation, that is, as in clinical settings, across days, and in physiological data.
The most important question to be answered in the clinical setting is: What specific treatment is effective with a specific patient and under what circumstances? The single-subject strategy is applicable whenever one seeks to isolate active components from a multiple-treatment therapy (Kendall \& Finch, 1978).

\section{REFERENCES}

Ax, A. (1981). Editorial. Biofeedback and Self Regulation. 6, 1-2. BANDERA, M., Bouchard, M., \& Granger, L. (1982). Voluntary control of autonomic responses: A case for a dialogue between individual and group experimental methodologies. Biofeedback and Self Regulation, 7, 317-329.

Bremner, F. J., McKenzie, R., Yost, M., \& Eddy, D. (1982). Interhemispheric EEG differences during relaxation, meditation, and biofeedback. Behavior Research Methods \& Instrumentation, 14, 300-302.

Breminer, F. J., Yost, M., \& MCKenzie, R. (1982). Computer managed inferential statistical analysis of EEG data. Behavior Research Methods \& Instrumentation, 14, 295-299.

Brown, G. A. (1982). The efficacy of single subject design in acute neurophysiological preparations. Unpublished master's thesis, Trinity University, San Antonio, TX.

Brown, G. A., \& Perez, F. I. (1985). Interfacing Apple and IBM computers for the analysis of clinical biofeedback. Behavior Research Methods, Instruments, \& Computers, 17, 165-167.

Carroll, D., \& PhYs-Davies, L. (1979). Heartrate changes with exercise and voluntary heartrate acceleration. Biological Psychology, 8 , 241-252.

HeRSEN, M., \& BARLow, D. (1976). Single case experimental designs: Strategies for studying behavior change. New York: Pergamon Press.

Johnston, J. M., \& Penny Packer, H. S. (1980). Strategies and tactics of human behavioral research. Hillsdale, NJ: Erlbaum.

KAZDIN, A. (1976). Statistical analysis for single-case experimental designs. In M. Hersden \& D. Barlow (Eds.), Single case experimental designs: Strategies for studying behavior change. New York: Pergamon Press.

Kelly, F. J., McNeil, K., \& Newman, I. (1973). Suggested inferential statistical models for research in behavior modification. Journal of Experimental Education, 41, 53-63.

Kendall, P. C., \& FinCH, A. J. (1978). Therapeutic biofeedback and the single subject strategy. Psychotherapy: Theory, Research, and practice, 15, 299-301.

Kewman, R. A. H., \& RoberTs, D. G. (1983). An alternative perspective on biofeedback efficacy studies. Biofeedback and Self Regulation, 8, 487-498.

Kolotkin, R. L., Billingham, K. A., \& Feldman, H. S. (1981). Computers in biofeedback research and therapy. Behavior Research Methods \& Instrumentation, 13, 532-542.

KRATOCHWILL, T. (1978). Single subject research: Strategies for evaluating change. New York: Academic Press.

SHINE, L. C. (1982). An illustration of how the effects of serial dependency are handled in single subject data. Educational and Psychological Measurement, 42, 87-94.

Steiner, S. S., \& DiNCE, W. M. (1983). A reply on the nature of biofeedback efficacy studies. Biofeedback and Self Regulation, 8, 499-503. 